Reprod. Nutr. Dévelop., 1980, 20 (5 A), 1415-1427.

\title{
Utilisation digestive comparée et formes d'excrétion fécale chez le rat, des acides érucique et brassidique estérifiés dans différentes structures glycéridiques
}

\author{
par J. P. SERGIEL, Lydia GABUCCI
}

Station de Recherches sur la Qualité des Aliments de l'Homme

I. N. R. A., 17, rue Sully, 21034 Dijon Cedex, France.

Summary. Digestibility and fecal excretion patterns of erucic and brassidic acids esterified in triglycerides of different structure. A comparative study in the rat.

The digestibility and fecal excretion patterns of erucic (cis n-9 docosenoic) and brassidic (trans n-9 docosenoic) acids, esterified in triglycerides of different structure, were compared in the rat. Synthetic triglycerides were prepared either by mixing or interesterifying trierucin or tribrassidin with an equal amount of corn oil. Rapeseed and peanut oils were used as references. The apparent digestibility of trierucin mixed or interesterified with corn oil was similar to that of peanut oil but higher than that of rapeseed oil. The high amount of 2-monoerucin resulting from diets containing trierucin explained the improved digestibility of erucic acid as compared to that of rapeseed oil which had a very small erucic acid content on the 2-position. It also seemed that 2-monoerucin enhanced the digestibility of saturated fatty acids. Tribrassidin mixed with corn oil had a poor digestibility because pancreatic lipase hydrolysis was delayed in the intestinal lumen. The high melting point of the homogeneous triglyceride was responsible for that phenomenon. The interesterification of tribrassidin with corn oil, which drastically lowered the melting point of the mixture from 52 to $37^{\circ} \mathrm{C}$, improved both the hydrolysis of the triglycerides and the digestibility of the C $22: 1$ trans fatty acid as well as that of the other fatty acids in the diets. Nevertheless, the unabsorbed brassidic acid in every case was principally excreted in the form of a compound insoluble in organic neutral solvent. In that respect, brassidic acid behaved like shorter chain saturated fatty acids such as palmitic and stearic acids.

\section{Introduction.}

Un certain nombre de travaux ont déjà été publiés ces dix dernières années sur l'utilisation digestive de l'acide érucique ou cis n-9 docosénoïque (C $22: 1)$ chez le Rat (Rocquelin et Leclerc, 1969 ; Rocquelin et al., 1975 ; Hornstra, 1972 ; Astorg et Rocquelin, 1975). Dans l'ensemble, les résultats obtenus montrent que cet acide gras à très longue chaîne est à l'origine de la médiocre utilisation digestive de certaines huiles de colza qui en contiennent jusqu'à 50 p. 100 des acides gras totaux. La respon- 
sabilité de ce phénomène n'incombe pas à une déficience de l'hydrolyse lipasique des triglycérides de l'huile (Sergiel et Rocquelin, 1975) mais elle est à mettre en relation avec leur structure glycéridique particulière qui est caractérisée par l'estérification préférentielle de l'acide érucique sur les positions 1 et 3 du glycérol. En effet, l'action de la lipase pancréatique sur de tels triglycérides donne naissance, dans la lumière intestinale, à de l'acide érucique libre qui est mal absorbé. L'interestérification de cette huile en répartissant le $C 22$ : 1 en proportions identiques entre les 3 positions du glycérol, entraîne une amélioration sensible de son utilisation digestive (Rocquelin ef al., 1971) et il en est de même lorsque cet acide gras est ingéré sous forme de triérucine (Martin, 1972). Dans ces deux derniers cas, l'amélioration de l'absorption de l'acide érucique est due à la présence, en forte proportion, de 2-monoérucine formée au cours de l'hydrolyse par la lipase pancréatique.

Par ailleurs, les huiles de colza ef de poisson partiellement hydrogénées, qui entrent dans la fabrication des margarines contiennent des proportions non négligeables d'isomères géométriques de monoènes à 22 atomes de carbone. L'acide brassidique, ou trans n-9 docosénoïque est l'un de ceux qui peuvent être formés à partir de l'huile de colza et sa digestibilité n'a fait l'objet, à ce jour, que de rares travaux (Rocquelin ef al., 1975 ; Astorg, à paraître).

Dans ce travail, nous avons comparé l'utilisation digestive des acides érucique ef brassidique en fonction de la structure glycéridique des matières grasses qui les renferment. Nous avons aussi approfondi l'analyse des différentes formes d'excrétion des lipides fécaux, afin notamment de vérifier indirectement si l'hydrolyse des triglycérides des régimes s'est déroulée de manière normale ou non pendant leur transit intestinal.

\section{Matériel et méthodes.}

\section{1) Traifement des animaux.}

Quarante-huit rats mâles de souche Wistar provenant de l'élevage EOPS ( ${ }^{1}$ ) de la Station, âgés de 3 semaines, et pesant en moyenne $65 \mathrm{~g}$ sont répartis en 6 lołs de 8 animaux. Chaque rat est mis dans une cage individuelle équipée d'un dispositif permettant de récolter séparément les fèces et les urines.

Les animaux reçoivent un régime semi-synthétique dont la composition détaillée est indiquée dans une précédente publication du laboratoire (Rocquelin ef al., 1969) et qui contient 15 p. 100 en poids ( 30 p. 100 en calories) de l'une des matières grasses suivantes : huile d'arachide, huile de colza riche en acide érucique, mélange triérucine $\left({ }^{2}\right)+$ huile de maïs $1 / 1(T E)$, mélange tribassidine $\left(^{3}\right)+$ huile de maïs $1 / 1(T B)$, triérucine interestérifiée avec l'huile de maïs (TEI) et tribrassidine interestérifiée avec l'huile de maïs (TBI). Dans ces deux derniers cas, les triglycérides homogènes sont interestérifiés avec l'huile de maïs dans le rapport $1 / 1$. La composition en acides gras

(1) E. O.P. S. = exempts d'Organismes Pathogènes Spécifiques.

(2) Triérucine = triglycéride homogène constitué d'acide érucique (cis $n-9$ docosénoïque).

$\left.{ }^{3}\right)$ Tribrassidine $=$ triglycéride homogène constitué d'acide brassidique (trans $n-9$ docosénoïque) 
de ces différentes matières grasses est rapportée dans le tableau 1 . Les régimes huile de colza, TE, TB, TEl et TBI ont des teneurs voisines en acides docosénoïques ( $C 22: 1$ ) cis ef trans.

\section{TABLEAU 1}

Composition en acides gras (p. 100 des esters méthyliques) ef températures de fusion des matières grasses des régimes

\begin{tabular}{|c|c|c|c|c|c|c|}
\hline & Arachide & Colza & TE & TEI & $\mathrm{TB}$ & $\mathrm{TBI}$ \\
\hline 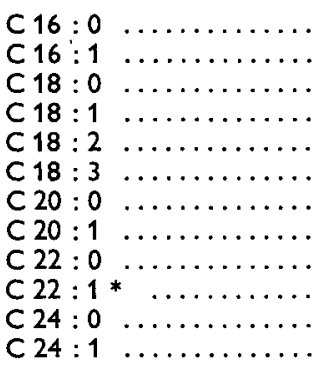 & $\begin{array}{c}10,5 \\
\operatorname{tr} . \\
3,1 \\
49,3 \\
28,5 \\
\operatorname{tr} . \\
1,9 \\
1,8 \\
2,8 \\
\operatorname{tr} . \\
1,3 \\
0,4\end{array}$ & $\begin{array}{c}3,6 \\
\text { tr. } \\
1,1 \\
12,3 \\
16,8 \\
10,1 \\
0,7 \\
6,3 \\
0,3 \\
47,4 \\
- \\
-\end{array}$ & $\begin{array}{r}6,5 \\
0,2 \\
1,2 \\
14,3 \\
31,3 \\
0,4 \\
0,4 \\
1,0 \\
0,5 \\
43,7 \\
- \\
-\end{array}$ & $\begin{array}{r}6,4 \\
0,1 \\
1,0 \\
14,8 \\
30,4 \\
0,5 \\
0,3 \\
1,0 \\
0,6 \\
44,6 \\
-\end{array}$ & $\begin{array}{r}6,2 \\
0,1 \\
1,1 \\
14,7 \\
31,9 \\
0,5 \\
0,5 \\
0,3 \\
1,0 \\
43,8 \\
-\end{array}$ & $\begin{array}{r}6,4 \\
0,1 \\
1,1 \\
15,2 \\
31,0 \\
0,5 \\
0,6 \\
0,3 \\
0,8 \\
43,9 \\
- \\
-\end{array}$ \\
\hline $\begin{array}{l}\text { Températures de fusion } \\
\text { totale } \ldots . . \ldots \ldots \ldots \ldots \ldots\end{array}$ & $10^{\circ} \mathrm{C}$ & $4^{\circ} \mathrm{C}$ & $24^{\circ} \mathrm{C}$ & $13^{\circ} \mathrm{C}$ & $52^{\circ} \mathrm{C}$ & $37^{\circ} \mathrm{C}$ \\
\hline
\end{tabular}

* Il s'agit du C $22: 1$ cis pour colza, TE et TEl et du $C 22: 1$ trans pour TB et TBI.

$\mathrm{TE}=$ Mélange de triérucine + huile de maïs $(1 / 1) ; \mathrm{TEI}=$ Mélange interestérifié de triérucine et d'huile de maïs $(1 / 1) ; T B=$ Mélange de tribrassidine + huile de maïs $(1 / 1) ; T B I=$ Mélange interestérifié de tribrassidine et d'huile de maïs $(1 / 1)$.

La nourriture est distribuée ad libitum et la quantité consommée par chaque animal est déterminée quotidiennement.

Les fèces collectées chaque jour sont entreposées dans des flacons étanches, au réfrigérateur, pendant la durée du bilan, puis conservées à $-20^{\circ} \mathrm{C}$ jusqu'à leur analyse. Les animaux sont pesés au début et à la fin de l'expérience qui dure 11 jours.

\section{2) Extraction des lipides fécaux.}

Les lipides fécaux sont extraits, rat par rat, par la méthode de Toullec et al. (1968) qui permet d'obtenir séparément la fraction soluble (FS) et la fraction insoluble (FI) dans le chloroforme méthanol 2/1 (V/V).

Une partie aliquote de la fraction soluble totale est saponifiée et les quantités d'acides gras de cette dernière ainsi que celles de la fraction insoluble sont déterminées par pesée.

Les différentes classes de lipides constitutives de la fraction soluble sont séparées par chromatographie sur couche mince de gel de silice. Les plaques, recouvertes de $0,5 \mathrm{~mm}$ de gel de silice G Merck, sont préparées selon la méthode de Stahl (1956), puis avant leur utilisation, elles sont « lavées » par développement dans le milieu chloroforme-méthanol-acide acétique $80: 25: 1$ (V/V). Les lipides de la fraction soluble, déposés en bande sur la plaque à l'aide d'un applicateur automatique d'échantillons, sont séparés par migration dans le milieu de développement constitué de : 
hexane-éther éthylique-méthanol-acide acétique $90: 20: 3: 2(\mathrm{~V} / \mathrm{V})$. Les différentes zones correspondant aux classes lipidiques (triglycérides, acides gras libres, diglycérides et monoglycérides) sont déłectées sous lumière ultraviolette après pulvérisation d'une solution éthanolique à 0,2 p. 100 de $2^{\prime} 7^{\prime}$ dichlorofluoresceine; le gel de silice est gratté et transvasé dans des flacons de $10 \mathrm{ml}$ à fermeture étanche téflon dans lesquels les lipides sont méthylés. Les acides gras de la fraction insoluble, de la fraction soluble totale et des différentes classes glycéridiques de cette dernière sont méthylés par le mélange benzène-méthanol à 14 p. 100 de trifluorure de bore $30: 70(\mathrm{~V} / \mathrm{V})$, à $90^{\circ} \mathrm{C}$ pendant $1 \mathrm{~h} 30$. L'addition d'une quantité connue d'heptadécanoate de méthyle (C $17: 0)$ à chacune des classes glycéridiques de la fraction soluble permet leur détermination quantitative.

Les analyses chromatographiques sont réalisées sur colonnes conventionnelles en acier inoxydable de $3 \mathrm{~m}$ de long et $2,1 \mathrm{~mm}$ de diamètre intérieur remplies de $5 \mathrm{p} .100$ de BDS (butanediol succinate) sur gas chrom Q 80/100 mesh. Ces colonnes sont utilisées en isotherme à $190^{\circ} \mathrm{C}$ dans des appareils équipés de détecteurs à ionisation de flamme. Le calcul de la composition centésimale en acides gras des échantillons, ainsi que la détermination de leur quantité sont effectués automatiquement par un intégrateurcalculateur Autolab System IV après programmation appropriée.

3) Analyse statistique des résultats.

Les grandeurs étudiées ont fait l'objel d'une analyse de variance (consommation de nourriture, gain de poids, efficacité alimentaire) ou de covariance (CUD) et les différentes moyennes ont été comparées au moyen du test $F$ de Fisher-Snedecor par la méthode des contrastes. Les calculs numériques ont été réalisés sur une calculatrice Olivetti P 602 à l'aide de programmes fournis par Lowy ef Manchon.

\section{Résulfats.}

1) Consommation de nourriture, gain de poids, efficacité alimentaire.

Les valeurs concernant ces différents paramètres sont rapportées dans le tableau 2.

TABLEAU 2

Consommation de nourriture, gain de poids et efficacité alimentaire pendant la durée de l'expérimentotion (11 jours) CUD apparent des acides gras totaux (moyennes \pm e. s. m. ; $n=8$ )

\begin{tabular}{|c|c|c|c|c|c|c|}
\hline & Arachide & Colza & TE & TEI & TB & TBI \\
\hline $\begin{array}{l}\text { Consommation de nour- } \\
\text { riture (g. de matière sè- } \\
\text { che) } \ldots \ldots \ldots \ldots \ldots \ldots \\
\text { Gain de poids }(g) \ldots \ldots \\
\text { Efficacité alimentaire } \\
\text { (gain de poids } / 100 \mathrm{~g} \\
\text { consommés) } \ldots \ldots \ldots \ldots \\
\text { CUD apparent des aci- } \\
\text { des gras totaux ....... }\end{array}$ & $\begin{array}{r}119,5 \pm 4,1^{a} \\
48,5 \pm 3,2^{a} \\
40,3 \pm 1,5 \\
95,1 \pm 0,3 b\end{array} \mid$ & $\mid \begin{array}{r}124,1 \pm 1,3 a \\
50,7 \pm 0,9 a \\
40,9 \pm 0,9 \\
92,8 \pm 0,6 c\end{array}$ & $\left\{\begin{array}{r}111,1 \pm 3,3 b \\
41,1 \pm 2,4 b \\
37,7 \pm 1,3 \\
95,3 \pm 0,3 b\end{array}\right.$ & $\mid \begin{array}{c}109,4 \pm 3,7 b \\
39,6 \pm 2,5 b \\
36,1 \pm 1,2 \\
96,2 \pm 0,3 a\end{array}$ & $\mid \begin{array}{r}125,8 \pm 3,1 \\
48,4 \pm 1,6 \\
\\
38,4 \pm 0,5 \\
73,1 \pm 1,3 d\end{array}$ & $\left\{\begin{array}{l}116,6 \pm 3,7 a \\
44,1 \pm 3,5^{a} \\
37,4 \pm 1,8 \\
82,0 \pm 1,6^{a}\end{array}\right.$ \\
\hline
\end{tabular}

Les moyennes affectées d'une même lettre ne diffèrent pas entre elles au seuil de $P=0,01$. 
a) Consommation de nourriture. - La comparaison entre la consommation de nourriture des animaux du lot arachide d'une part, et celle de tous les autres lots ingérant du $C 22$ : 1 cis ou trans d'autre part, ne montre pas de différence statistiquement significative. On remarque néanmoins, en comparant entre eux les régimes qui renferment des acides gras en $C 22: 1$, que ceux qui apportent l'acide érucique sous forme de triglycérides de synthèse (TE et TEl) entraînent un abaissement faible mais significatif de la consommation de nourriture, par rapport à ceux qui contiennent de l'acide brassidique (TB ef TBI) ou de l'huile de colza.

b) Gain de poids ef efficacité alimentaire. - Au niveau des gains de poids, les différences vont dans le même sens que celles qui ont été relevées pour la consommation de nourriture.

Les efficacités alimentaires ne sont pas significativement différentes.

2) Coefficients d'utilisation digestive apparente (CUD) des acides gras.

Les CUD des acides gras totaux des régimes TE ef TEl sont les plus élevés, et ils se situent à un niveau égal, voire supérieur à celui des acides gras totaux de l'huile d'arachide (tabl. 2). La tribrassidine et la tribrassidine interestérifiée donnent des valeurs nettement diminuées par rapport aux autres matières grasses. L'interestérification améliore le CUD de la triérucine et de la tribrassidine avec un effet plus marqué pour cette dernière. L'huile de colza a un CUD intermédiaire entre les 2 groupes de valeurs précédentes.

Dans le tableau 3 figurent les CUD de chacun des acides gras dont la teneur dans le régime est supérieure à 1 p. 100. Pour les autres, il est difficile d'établir un CUD valable à cause des variations importantes des quantités excrétées.

TABLEAU 3

Coefficient d'utilisation digestive apparente de chaque acide gros des régimes

\begin{tabular}{|c|c|c|c|c|c|c|}
\hline & Arachide & Colza & TE & TEI & $\mathrm{TB}$ & TBI \\
\hline 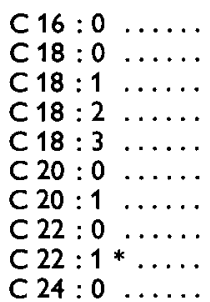 & $\begin{array}{c}91,3 \pm 0,4 a \\
72,5 \pm 1,8 a \\
98,8 \pm 0,1 a \\
99,8 \pm 0,02 \\
-1 \pm 2,7 \\
60,9 \pm 2,3 \pm 0,4 \\
93,3 \pm 0,0 \\
59,0 \pm 2,9 \\
43,6 \pm 4,1\end{array}$ & $\begin{array}{c}94,3 \pm 0,4 b \\
85,1 \pm 1,1 b \\
98,0 \pm 0,2 b \\
99,6 \pm 0,04 \\
99,9 \pm 0,01 \\
91,2 \pm 0,8 \\
89,0 \pm 1,0 a\end{array}$ & $\begin{array}{c}97,6 \pm 0,3 c \\
92,0 \pm 0,5 c \\
99,2 \pm 0,1^{c} \\
99,9 \pm 0,02 \\
\pm \\
95,8 \pm 0,5 \\
90,7 \pm 0,6 a \\
\pm\end{array}$ & $\begin{array}{c}97,5 \pm 0,3 c \\
92,0 \pm 0,9 c \\
98,7 \pm 0,6 a \\
99,9 \pm 0,01 \\
\pm \\
96,5 \pm 0,5 \\
92,9 \pm 0,6 b \\
-0\end{array}$ & $\begin{array}{c}80,6 \pm 1,2 d \\
70,4 \pm 1,8 a \\
94,9 \pm 0,8 b \\
98,9 \pm 0,2 \\
= \\
= \\
45,7 \pm 2,9 c \\
=\end{array}$ & $\begin{array}{c}86,0 \pm 1,3 e \\
66,4 \pm 2,9 a \\
96,7 \pm 0,3 b \\
99,2 \pm 0,1 \\
= \\
= \\
64,4 \pm 3,2 a\end{array}$ \\
\hline
\end{tabular}

* II s'agit du C $22: 1$ cis pour colza, TE et TEl et du C $22: 1$ trans pour TB ef TBI.

D'une manière générale, les acides gras insaturés à 18 atomes de carbone ont un CUD très élevé qui varie entre 98 ef 100 p. 100 . On note toutefois dans les lots TB et TBI une tendance à l'affaiblissement du CUD du C $18: 1$ dont les valeurs sont respectivement de 94,7 et 96,7 p. 100. 
Les CUD des acides gras saturés $C 16: 0$ et $C 18$ : 0 sont significativement plus élevés chez les animaux des lots colza, TE et TEI par rapport à ceux qui reçoivent les autres régimes. Les acides gras saturés à chaîne plus longue (C $20: 0, C 22: 0$, C $24: 0)$ présents dans l'huile d'arachide sont relativement mal absorbés.

Le CUD du C $22: 1$ cis est nettement supérieur à celui du C $22: 1$ trans, mais l'interestérification améliore sensiblement la digestibilité de ce dernier.

3) Lipides fécaux.

a) Quantités d'acides gras excrétés dans la fraction soluble (FS) ef dans la fraction insoluble (FI) (tabl. 4). - Chez les animaux ingérant de l'acide érucique (lots colza,

TABLEAU 4

Quantités d'acides gras excrétés dans la fraction soluble (FS) et dans la fraction insoluble (FI) des lipides fécaux, pendant la durée de l'expérimentation (moyennes \pm e. $\mathbf{s .} \mathbf{m} . ; \mathbf{n}=\mathbf{8}$ )

\begin{tabular}{|c|c|c|c|c|c|c|}
\hline & Arachide & Colza & TE & TEI & TB & TBI \\
\hline $\begin{array}{l}\text { Acides gras excrétés } \\
\text { dans la FS (mg) ....... } \\
\text { Acides gras excrétés } \\
\text { dans la Fl }(\mathrm{mg}) \ldots \ldots \\
\frac{\mathrm{FS}}{\mathrm{FS}-\mathrm{Fl}}(\mathrm{p} 100) \ldots \ldots\end{array}$ & $\begin{array}{c}99 \pm 9,6 \\
718 \pm 62,7 \\
12,1\end{array}$ & $\begin{array}{c}535 \pm 59,6 \\
720 \pm 97,8 \\
42,6\end{array}$ & $\begin{array}{c}407 \pm 39,8 \\
306 \pm 50,7 \\
57,1\end{array}$ & $\begin{array}{c}245 \pm 33,2 \\
348 \pm 40,4 \\
41,3\end{array}$ & $\begin{array}{c}500 \pm 69,3 \\
4232 \pm 260,1 \\
10,6\end{array}$ & $\begin{aligned} & 274 \pm 52,3 \\
& 2647 \pm 248,4 \\
& 9,4\end{aligned}$ \\
\hline
\end{tabular}

TE ef TEI) les acides gras totaux excrétés sont en gros répartis également entre la FS et la FI. En effet, pour les lots colza et TEI, la proportion de FS par rapport aux acides gras totaux est voisine de 42 p. 100 , et pour le lot TE, elle est de 57 p. 100. Avec les régimes qui contiennent de l'acide brassidique (TB et TBI) les acides gras de la FS ne représentent que 10 p. 100 des acides gras fécaux. Ceffe proportion reste constante malgré les différences observées au niveau des quantités d'acides gras totaux excrétés qui sont, pour le lot TBI inférieures de 45 p. 100 à celles du lot TB. Avec l'huile d'arachide, la proportion de FS (12 p. 100) est très proche de celle obłenue avec les régimes TBI ef TB mais la quantité d'acides gras excrétés est 4 à 6 fois plus faible.

b) Composition en acides gras de la fraction insoluble et de la fraction soluble totale (tabl. 5). - Dans le lot arachide, la FS se caractérise par une teneur élevée en C 16:0 (21 p. 100) et en C $18: 1$ (44 p. 100). Par contre, la Fl, qui est quantitativement la plus importante fait apparaître, par rapport aux acides gras ingérés et à la FS un fort enrichissement en acides gras saturés, particulièrement marqué pour le $C 22: 0$ ef le C 24 : 0 . Chez les animaux soumis à des régimes contenant de l'acide érucique (lots colza, TE et TEI), les compositions en acides gras des fractions soluble et insoluble sont très voisines et l'acide érucique représente entre 75 et 86 p. 100 des acides gras totaux. La Fl est toujours un peu plus riche que la FS en acides gras saturés à longueur de chaîne supérieure ou égale à 18 atomes de carbone. Les fractions solubles des lots $T B$ et $T B I$ sont légèrement différentes ; celle du lot TB présente une teneur plus élevée en $C 22: 1$ trans qui s'accompagne d'une diminution du $C 16: 0$ et du $C 18: 1$. Les 


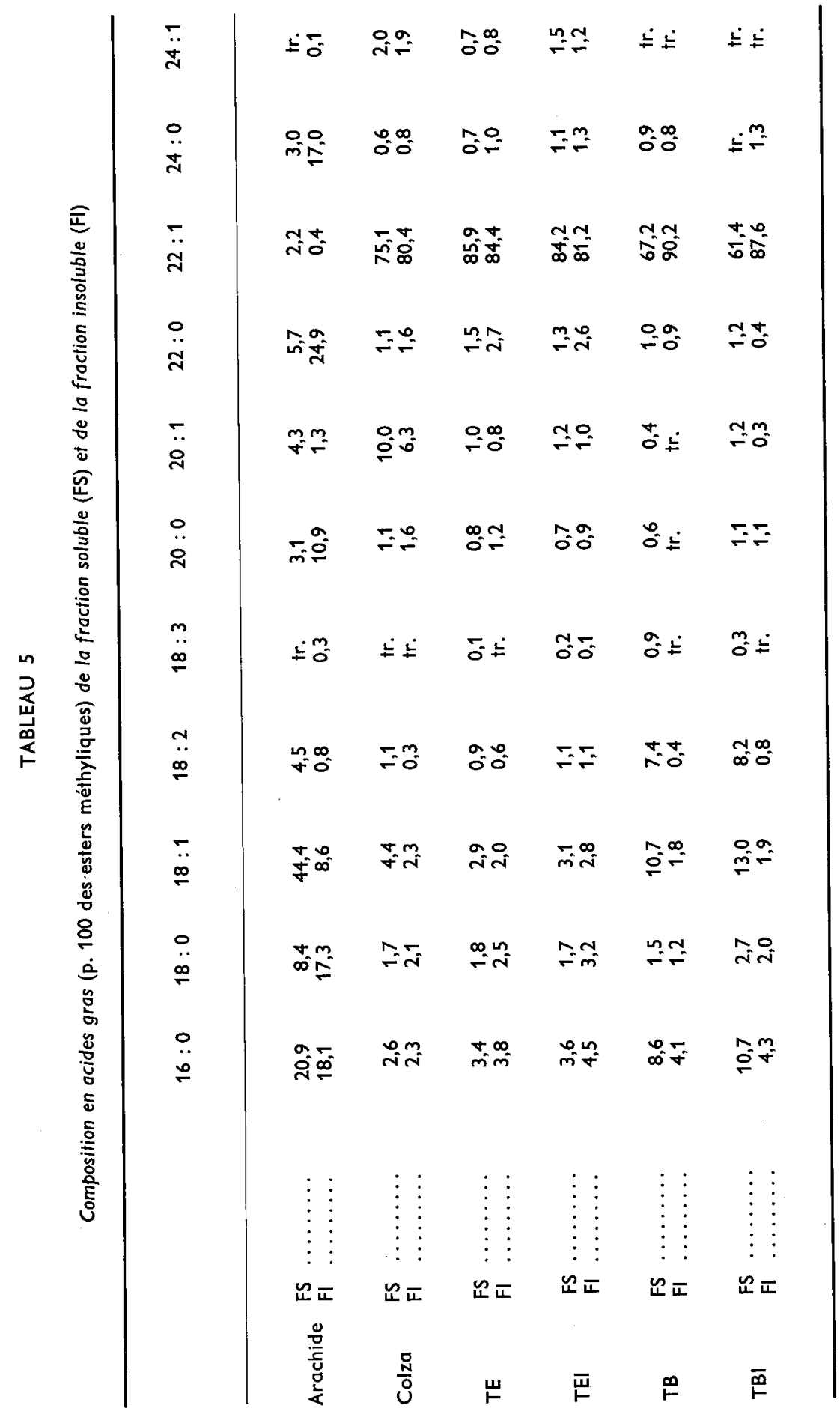


fractions insolubles, dont les compositions sont très voisines dans les lots TB et TBI contiennent jusqu'à 90 p. 100 d'acide brassidique.

c) Composition lipidique de la fraction soluble. - Les résultats relatifs à la répartition des différentes classes lipidiques dans la fraction soluble, à savoir : triglycérides (TG), acides gras libres (AGL), diglycérides (DG) ef monoglycérides (MG), ainsi que la composition en acides gras de chacune d'elles, sont rapportés dans le tableau 6.

En règle générale, les acides gras libres constituent de très loin, la classe prépondérante (88 à 96 p. 100) sauf dans le cas du lot TB où ils n'atteignent que 60 p. 100 du total. Les autres classes lipidiques - sauf dans le lot TB - représentent 4 à 12 p. 100 de l'ensemble des acides gras et leur répartition est très variable. Ainsi, les monoglycérides qui dans certains cas n'apparaissent qu'à l'état de traces difficilement décelables en chromatographie sur couche mince, s'élèvent à 2,6 p. 100 dans les lots TE et TEl. Les diglycérides sont présents dans tous les cas en faibles proportions ( 0,6 d 5,6 p. 100). Les triglycérides, sauf dans le lot TB où ils atteignent 37 p. 100 , sont eux aussi peu abondants.

La composition en acides gras de la classe des AGL montre dans presque tous les cas une similitude nette avec celle de la fraction soluble totale et ceci s'explique aisément étant donné que cette dernière est constituée en majeure partie d'acides gras non estérifiés.

Le cas des triglycérides de la fraction soluble du lot TB est intéressant à mentionner, car avec 82,4 p. 100 d'acide brassidique, on peut considérer qu'ils renferment une forte proportion de tribrassidine provenant des triglycérides homogènes ingérés et non hydrolysés.

Dans tous les autres lots, les compositions en acides gras des triglycérides, diglycérides et monoglycérides - dans la mesure où ils existent - présentent par rapport à celles des lipides alimentaires un enrichissement en acides gras saturés à 16 et 18 atomes de carbone et aussi en chaînes à 20,22 et $24 \mathrm{C}$ dans le cas de l'arachide. Par ailleurs, avec tous les régimes confenant du $C 22$ : 1 cis ou trans, on observe une proportion élevée de ce dernier dans les glycérides fécaux.

\section{Discussion.}

Dans cetfe expérience, deux effets principaux sont à considérer : d'une part, ceux dús à la présence, dans les matières grasses ingérées, d'acides gras monoinsaturés à très longue chaîne (22 atomes de carbone) sous deux formes isomériques cis et trans, d'autre parf, les effets liés à l'incorporation de ces acides gras, en proportions égales, dans des structures glycéridiques par ailleurs différentes (triglycérides mixtes d'huile, triglycérides homogènes mélangés ou interestérifiés avec des triglycérides mixtes d'huile de maïs).

Au niveau des paramètres tels que consommation de nourriture, gain de poids et efficacité alimentaire, nos résultats laissent apparaître des différences peu accusées. On remarque simplement, avec les triglycérides de synthèse, que les animaux qui ingèrent les acides gras trans, ont une consommation de nourriture plus importante que ceux qui sont nourris avec des acides gras cis. Cette constatation est en accord avec celles de Rocquelin et al. (1975) et Astorg et al. (à paraître). L'huile de colza, composée 


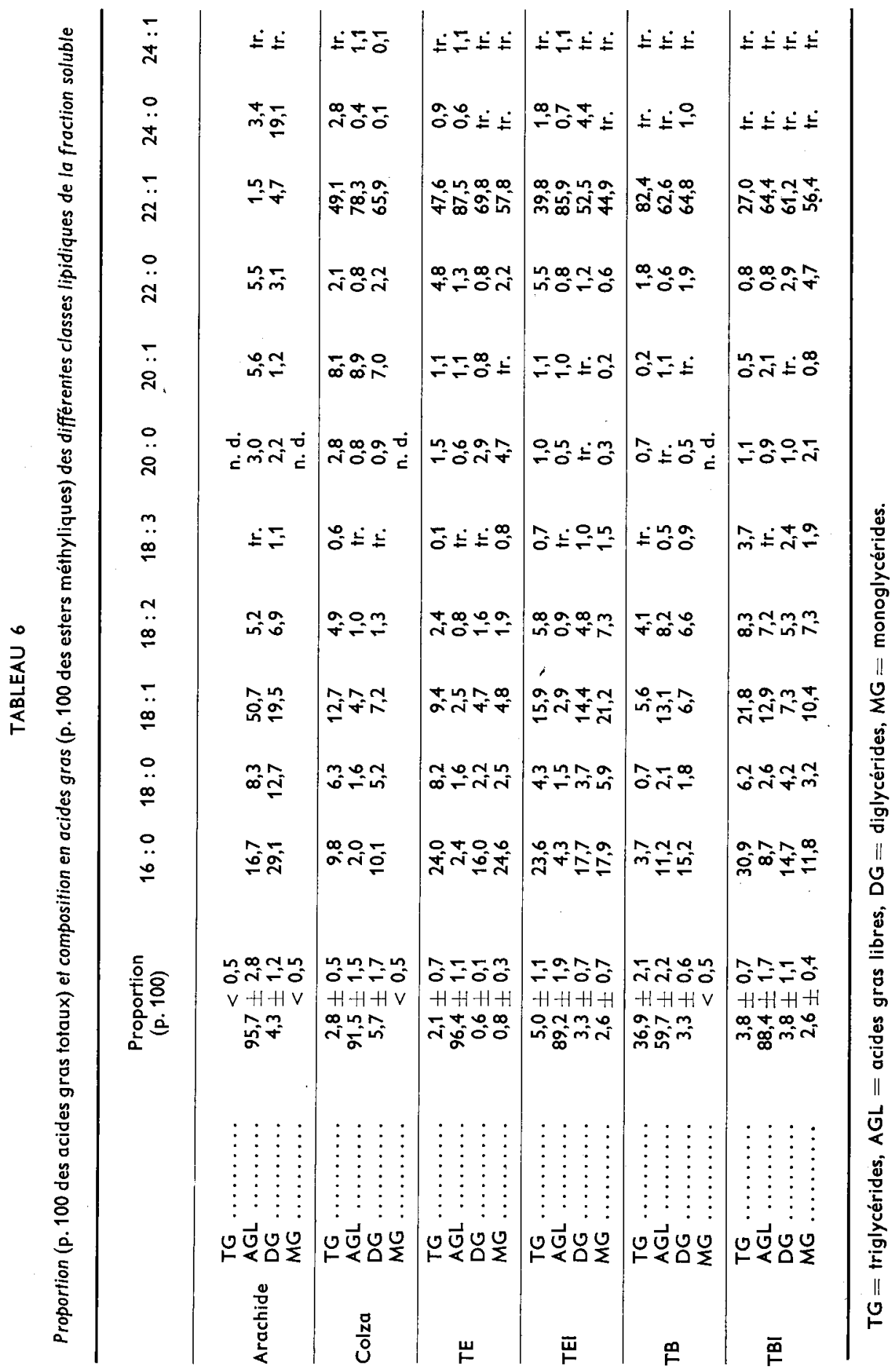


de triglycérides mixtes dans lesquels l'acide érucique est préférentiellement estérifié sur les positions 1 et 3 , n'affecte de façon défarorable, ni la consommation de nourriture, ni le gain de poids par rapport à l'huile d'arachide ou aux triglycérides de synthèse.

Les triglycérides de synthèse TE et TEI ont un CUD plus élevé que celui de l'huile de colza, et comparable à celui de la plupart des huiles alimentaires courantes. Cette augmentation du CUD est due à un accroissement de la digestibilité de tous les acides gras mais surtout du $C 16: 0$ et du $C 18: 0$. L'interestérification ne modifie pratiquement pas le CUD tolal des acides gras et n'augmente que très peu celui du C $22: 1$. Ce résultat peut s'expliquer, si l'on considère que globalement et uniquement en ce qui concerne l'acide érucique, la structure glycéridique des deux matières grasses TE et TEI, c'est-à-dire la répartition relative du C 22 : 1 sur les positions 1, 2 et 3 du glycérol est identique. Etant donné que l'on ne retrouve que très peu de triglycérides dans les lipides fécaux des lots TE et TEI, on peut admettre que leur hydrolyse lipasique est pratiquement complète, et que, au moment de l'absorption, il y a pour chacun d'eux, autant de 2 monoérucine et d'acide érucique libérés dans la lumière intestinale. L'utilisation digestive plus complète des acides gras totaux des lots TE et TEI, par rapport à celle des huiles de colza et d'arachide, est vraisemblablement due, non seulement à la bonne absorption de la 2 monoérucine en tant que telle, mais aussi à son influence bénéfique sur la résorption des autres acides gras libres. En outre, les valeurs nettement plus élevées des CUD du C $16: 0$ et du $C 18: 0$ des lots TE et TEl, par rapport à celles du lot arachide, semblent indiquer que la 2-monoérucine a un effet bénéfique sur l'absorption des chaînes saturées, plus marqué que celui de la monooléine formée à partir de l'huile d'arachide. Cette interprétation est en accord avec des résultats obtenus par Martin (1972) qui faif état de CUD plus élevés des acides palmitique et stéarique lorsqu'ils sont en mélange avec de la triérucine, que lorsqu'ils sont en présence de proportions identiques de trioléine. Un travail récent de Astorg et al. (à paraître) va aussi dans ce sens. Le mécanisme mis en jeu pourrait se situer au niveau d'un effet inhibiteur plus marqué de la monoérucine que de la monooléine sur la formation de complexes insolubles, ou d'une action favorable sur la solubilisation micellaire des acides gras saturés. Il serait intéressant de vérifier ces hypothèses car elles suggèrent que les caractéristiques physicochimiques du milieu intestinal, qui conditionnent l'absorption des lipides peuvent varier non seulement selon l'apport qualitatif et quantitatif des acides gras, mais aussi en fonction de l'association de ces derniers à des structures glycéridiques particulières.

Deux causes principales peuvent être invoquées pour expliquer le faible CUD des acides gras totaux dans le lof tribrassidine + maïs : d'une part, une hydrolyse lipasique incomplète de la tribrassidine, très vraisemblablement à cause de son point de fusion élevé $\left(57^{\circ} \mathrm{C}\right)$ et d'autre part, une mauvaise absorption de l'acide brassidique libéré, pour des raisons qui ne sont pas toutes connues, mais dont la principale est la formation de complexes insolubles, qui représentent 90 p. 100 des acides gras totaux excrétés. En fait, nous avons constaté un déficit d'hydrolyse de la tribrassidine alimentaire, mais il ne suffit pas à lui seul pour expliquer le faible CUD des acides gras totaux. En effet, la tribrassidine non hydrolysée ne représente que 3,8 p. 100 de l'ensemble des acides gras fécaux et n'intervient que pour 0,8 p. 100 dans la valeur du CUD des acides gras totaux. On peut alors penser que, par suite du ralentissement de son 
hydrolyse, la tribrassidine du régime serait dégradée dans des zones distales de l'intestin où l'absorption est moins active, ce qui favoriserait la formation de complexes insolubles.

L'hydrolyse retardée de la tribrassidine peut aussi expliquer les valeurs abaissées du CUD des autres acides gras du régime TB + maïs par un effet d'entraînement des acides gras ou des triglycérides issus de l'huile de maïs, dans un bol alimentaire dont les caractéristiques physicochimiques ne correspondent pas à un optimum en matière de digestion et d'absorption.

L'interestérification de la tribrassidine avec l'huile de maīs (cas du lot TBI) en modifiant la composition et la structure glycéridiques de la matière grasse, abaisse sa température de fusion de $52^{\circ} \mathrm{C}$ à $37^{\circ} \mathrm{C}$. Ce phénomène entraîne sans doute des changements dans la cinétique et le rendement de l'hydrolyse lipasique, car on ne retrouve pratiquement plus de triglycérides dans les fèces. II en résulte une nette augmentation du CUD de l'acide brassidique qui passe de 45,7 à 64,4 p. 100. Comme la proportion de fraction soluble par rapport aux acides gras tolaux excrétés reste constante dans les lots $\mathrm{TB}$ et $\mathrm{TBI}$, on peut donc considérer que l'absorption moins complète de l'acide brassidique, comparativement à celle de l'acide érucique est due à son élimination préférentielle sous forme de complexes insolubles.

De ce dernier point de vue, on constate que l'isomérie trans, confère à l'acide brassidique des propriétés proches de celles des acides gras saturés à chaîne plus courte (palmitique et stéarique) qui eux sont excrétés sous forme de savons de calcium (Flanzy et al., 1968 ; Fakambi, 1970). Comme ces deux types d'acides gras ont un point de fusion élevé voisin de $60^{\circ} \mathrm{C}$, on peut admettre que cette caractéristique, qui est liée à la configuration de ces molécules, est l'un des facteurs responsables de leur élimination sous forme de complexes insolubles. Le niveau d'action d'un tel facteur sur les processus de digestion et d'absorption des lipides n'est pas connu, mais des travaux in vitro ont mis en évidence une difficulté de solubilisation micellaire des acides palmitique et stéarique (Savary et Constantin, 1966) et une affiniłé de ces derniers, vis-à-vis du calcium, beaucoup plus forte que celle des acides gras insaturés à point de fusion nettement plus bas (Toullec ef al., 1968). On peut supposer que le $C 22: 1$ trans a un comportement similaire.

Nos résultats relatifs à la composition en acides gras des glycérides de la fraction soluble, font apparaître dans ces derniers, une augmentation systématique de la teneur en acides gras saturés (palmitique et stéarique surtout) par rapport à celle des triglycérides du régime. Ceci suggère, qu'en plus de la lipolyse, il se produit au niveau de l'intestin, des réactions d'interestérification ou d'estérification entre les triglycérides ou les glycérides partiels et les acides gras libres. Un tel phénomène a été mis en évidence par Borgström (1954). En ce qui nous concerne, les acides palmitique et stéarique qui participent à ces processus peuvent être d'origines diverses : à ceux qui dérivent du régime viennent probablement s'ajouter ceux qui proviennent de l'apport lipidique endogène ef ceux issus de la biohydrogénation intestinale des acides gras insaturés. Toułefois, cetłe dernière éventualité d'une formation des acides gras saturés par biohydrogénation est à exclure car il n'y a pratiquement pas de C 16 : 1 dans nos régimes, ef l'autre preuve de l'absence d'une telle réaction est fournie par les animaux qui ingèrent du $C 22: 1$ et chez lesquels on ne retrouve pas au niveau fécal de quantités appréciables de C 22 : 0 . Quant à la part qui revient à l'apport 
endogène, elle est difficile à estimer, mais certains auteurs (Flanzy et al., 1968) ont montré par exemple chez le Porc, que les lipides fécaux d'un régime lipidoprive renferment 34 p. 100 de $C 16: 0$ et 41 p. 100 de $C 18: 0$. De toułe façon, étant donné les faibles quantités de glycérides excrétées dans les fèces, ces processus d'estérification sélective des acides gras saturés, ne contribuent que pour une part infime à l'abaissement de leur CUD.

En conclusion, nos résultats tendent à confirmer que des triglycérides de synthèse comportant de l'acide érucique, sont presque complètement hydrolysés pendant leur transit intestinal quelle que soif leur structure. D'autre part, quand de tels triglycérides contiennent la proportion maximale possible d'acide érucique estérifié en position 2, l'utilisation digestive apparente des acides gras totaux est meilleure que lorsque cet acide gras est réparti sur les positions 1 et 3, l'amélioration portant à la fois sur les acides gras saturés et sur l'acide érucique. L'interestérification de la triérucine avec l'huile de maïs accroît le CUD de l'acide érucique, par rapport à celui obtenu avec l'huile de colza et avec la triérucine, mais l'effet est moins marqué que celui observé par Rocquelin ef al. (1971) qui comparaient une huile de colza normale à la même huile interestérifiée.

La faible absorption de l'acide brassidique présent en proportion élevée (44 p. 100) dans une matière grasse sous forme de triglycéride homogène (tribrassidine) est en partie liée à l'hydrolyse intraluminaire incomplète de ce dernier. L'interestérification de la tribrassidine avec une quantité égale d'huile de maïs, en abaissant la température de fusion du mélange de triglycérides de $52^{\circ} \mathrm{C}$ à $37^{\circ} \mathrm{C}$, entraîne une amélioration de l'hydrolyse lipasique intestinale des triglycérides ef augmente nettement l'utilisation digestive de l'acide brassidique. Cet acide gras est toutefois plus faiblement absorbé que l'acide érucique et il est toujours principalement excrété sous forme de complexes insolubles. Ceci suggère que le $C 22: 1$ trans, à cause de son point de fusion élevé, lié à sa configuration stérique, $a$, au plan des phénomènes de digestion et d'absorption, des propriétés voisines de celles des acides gras saturés (C $16: 0$ et $C 18: 0)$ dont les points de fusion lui sont comparables.

Reçu en juin 1979. Accepté en mars 1980.

Remerciements. - Nous remercions la Société Astra-Calvé qui nous a fourni gratuifement les triglycérides de synthèse et qui a effectué leur interestérification avec l'huile de maïs, ainsi que $M$. Suschetet pour sa collaboration à l'analyse statistique des résultats.

\section{Références}

ASTORG P. O., ROCQUELIN G., 1975. Utilisation digestive d'une huile de hareng partiellement hydrogénée chez le rat. Ann. Biol. anim. Bioch. Biophys., 15, 611-614.

BORGSTROM B., 1954. The formation of new glyceride ester bonds during digestion of glycerides in the lumen of the small intestine of the rat. Arch. Biochem. Biophys., 49, 268-275.

FAKAMBI L., 1970. Interaction du calcium et des lipides alimentaires : mise en évidence de l'excrétion fécale de savons de calcium chez le rat. Th. Doct. $3^{e}$ Cycle, Fac. Sci. Paris.

FLANZY J., RÉRAT A., FRANÇOIS A. C., 1968. Etude de l'utilisation digestive des acides gras chez le porc. Ann. Biol. anim. Bioch. Biophys., 8, 537-548. 
HORNSTRA G., 1972. Digestibility, efficiency and other metabolic effects of dietary rapeseed oil in rats. Nutr. Metabol., 14, 282-297.

MARTIN B., 1972. Influence des huiles de colza et de canbra, et de mélanges de triglycérides homogènes reproduisant certaines caractéristiques de ces huiles sur les lipides hépatiques du rat. Th. Doct. 3e Cycle, Univ. Dijon.

ROCQUELIN G., JUANEDA P., PELERAN J. C., ASTORG P. O., 1975. Effets comparés, à court terme, des acides n-9 trans docosénoïque (brassidique) et n-9 cis docosénoïque (érucique) sur les lipides cardiaques du rat sevré. Nutr. Metabol., 19, 113-126.

ROCQUELIN G., LECLERC J., 1969. L'huile de colza riche en acide érucique ef I'huile de colza sans acide érucique. II. - Utilisation digestive comparée chez le rat. Ann. Biol. anim. Bioch. Biophys., 9, 413-426.

ROCQUELIN G., SERGIEL J. P., MARTIN B., LECLERC J., CLUZAN R., 1971. The nutritive value of refined rapeseed oils. A review. J. Amer. Oil Chemists'Soc., 48, 728-732.

SAVARY P., CONSTANTIN M. J., 1966. Sur la résorption intestinale des chaînes éruciques et leur incorporation dans les chylomicrons lymphatiques du rat. Biochim. biophys. Acło, 125, 118-128.

SERGIEL J. P., ROCQUELIN G., 1975. Digestibilité des huiles de colza, de canbra et d'arachide. Etude de leur lipolyse « in vitro » et « in vivo ». Ann. Biol. anim. Bioch. Biophys., 15, 103-114.

STAHL E., 1956. Dünnschicht Chromatographie. Pharmazie, 11, 633-637.

TOULLEC R., FLANZY J., RIGAUD J., 1968. Dosage des lipides des fèces. Extraction séparée, importance et composition en acides gras des lipides non saponifiés et de ceux des complexes insolubles. Ann. Biol. anim. Bioch. Biophys., 8, 281-289. 